\section{(6) OPEN ACCESS}

RESEARCH PAPER

\title{
Attention! A good bedside test for delirium?
}

\author{
Niamh A O'Regan, ${ }^{1}$ Daniel J Ryan, ${ }^{1}$ Eve Boland ${ }^{2}$ Warren Connolly, ${ }^{2}$ Ciara McGlade, \\ Maeve Leonard, ${ }^{3}$ Josie Clare, ${ }^{4}$ Joseph A Eustace, ${ }^{5}$ David Meagher, ${ }^{6,7}$ \\ Suzanne Timmons ${ }^{1}$
}

\begin{abstract}
- Additional material is published online only. To view please visit the journal online (http://dx.doi.org/10.1136/ jnnp-2013-307053).

For numbered affiliations see
\end{abstract} end of article

\section{Correspondence to}

Dr Niamh O'Regan, Centre for Gerontology and

Rehabilitation, School of Medicine, University College Cork, St Finbarr's Hospital, Douglas Road, Cork, Ireland; niamhoregan78@yahoo.co.uk

Received 18 October 2013 Revised 18 January 2014 Accepted 22 January 2014 Published Online First 25 February 2014

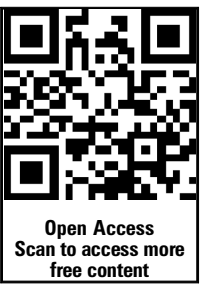

CrossMark

To cite: $O^{\prime}$ Regan NA, Ryan DJ, Boland $\mathrm{E}$, et al. I Neurol Neurosurg Psychiatry 2014;85: 1122-1131.

\section{ABSTRACT}

Background Routine delirium screening could improve delirium detection, but it remains unclear as to which screening tool is most suitable. We tested the diagnostic accuracy of the following screening methods (either individually or in combination) in the detection of delirium: MOTYB (months of the year backwards); SSF (Spatial Span Forwards); evidence of subjective or objective 'confusion'. Methods We performed a cross-sectional study of general hospital adult inpatients in a large tertiary referral hospital. Screening tests were performed by junior medical trainees. Subsequently, two independent formal delirium assessments were performed: first, the Confusion Assessment Method (CAM) followed by the Delirium Rating Scale-Revised 98 (DRS-R98). DSM-IV (Diagnostic and Statistical Manual of Mental Disorders, fourth edition) criteria were used to assign delirium diagnosis. Sensitivity and specificity ratios with 95\% Cls were calculated for each screening method.

Results 265 patients were included. The most precise screening method overall was achieved by simultaneously performing MOTYB and assessing for subjective/objective confusion (sensitivity $93.8 \%, 95 \% \mathrm{Cl} 82.8$ to 98.6 ; specificity $84.7 \%, 95 \% \mathrm{Cl} 79.2$ to 89.2$)$. In older patients, MOTYB alone was most accurate, whereas in younger patients, a simultaneous combination of SSF (cutoff 4) with either MOTYB or assessment of subjective/ objective confusion was best. In every case, addition of the CAM as a second-line screening step to improve specificity resulted in considerable loss in sensitivity.

Conclusions Our results suggest that simple attention tests may be useful in delirium screening. MOTYB used alone was the most accurate screening test in older people.

\section{INTRODUCTION}

Delirium is a serious neuropsychiatric condition which occurs in the setting of acute illness. It is ubiquitous in the acute hospital setting, having a point prevalence of almost $20 \%,{ }^{1}$ with higher rates in older patients. It is independently associated with adverse outcomes, ${ }^{2}$ including increased length of stay, increased mortality and accelerated cognitive and functional decline.

A major challenge in delirium care is that, despite its significance, delirium is commonly missed or mistaken for other conditions across treatment settings. In a point-prevalence study of delirium in 311 patients across a general hospital, doctors missed over half the delirium cases. ${ }^{1}$ In a study of general hospital referrals to liaison psychiatry, Kishi et al showed that $46 \%$ of delirium diagnoses were missed by the referring team. ${ }^{3}$ Detection rates are lower in older patients, ${ }^{4}$ those with premorbid dementia ${ }^{5}$ and in hypoactive cases. ${ }^{67}$ Collins et al found recognition rates to be as low as $28 \%$ in older medical inpatients ${ }^{8}$ and studies in the emergency department (ED) show similar rates of underdetection. ${ }^{9}{ }^{10}$ The reasons for poor recognition are multifactorial. 'Confusion' is commonly considered normal in older patients, who are most at risk. The symptom profile varies greatly from patient to patient, and the prevailing stereotype of hyperactive delirium ('delirium tremens') is misleading, as delirium most commonly presents in its less obvious and more serious hypoactive form. Clinicians may also be deceived by patients during periods of lucidity, as the symptoms characteristically fluctuate over the course of the day. Studies have shown the importance of early recognition and intervention in reducing the severity and duration of delirium. ${ }^{11}$ Although studies investigating impact of early intervention on long-term outcomes have been inconsistent, Gonzalez and colleagues found an $11 \%$ increase in mortality with every additional $48 \mathrm{~h}$ of delirium, ${ }^{12}$ and Kakuma et $a l^{13}$ reported a significant increase in mortality at 6 months in older patients with undetected delirium discharged from the ED, compared to those with delirium who had been appropriately diagnosed.

Ideally, all patients at high risk of delirium should be assessed regularly using systematic application of sensitive tools. However, formal delirium diagnosis is based on thorough, and often lengthy, assessment by a trained and experienced clinician. Hence, a two-phase approach to detection is most efficient: first, screening for key delirium features using a simple, short test, followed by formal assessment in those who screen positive. The recent National Institute of Health and Care Excellence (NICE) guidelines advocate this approach, ${ }^{14}$ and recommend daily screening for all those at risk. Currently, consensus is lacking as to which screening method is best, but it is clear that test sensitivity must be emphasised over specificity to minimise the dangers of missed cases. The NICE guidelines screening approach is based on monitoring for a list of specific delirium indicators, however, this method has yet to be validated and likely requires some understanding of delirium in order to ensure accurate application. The most widely used screening test is the Confusion Assessment Method $(\mathrm{CAM})^{15}{ }^{16}$ which has been validated in several languages and settings. ${ }^{17}$ This tool, designed for delirium diagnosis, was based on DSM-IIIR (Diagnostic and Statistical Manual of Mental Disorders, third 
edition) criteria. It requires training to ensure accuracy ${ }^{18}$ and lacks the brevity desired for routine general use.

The prevailing gold standard for delirium diagnosis at the time of the study was DSM-IV ${ }^{19}$ criteria applied by an experienced clinician following standardised testing. In order to be diagnosed with DSM-IV delirium, a patient must present with the following features: a disturbance of consciousness with reduced ability to focus, shift or sustain attention; a change in cognition or perception that is not explained by a pre-existing, established or evolving dementia; acute onset and fluctuating course; and evidence of an underlying general medical cause. Hence, inattention is a core delirium feature, mandatory to DSM-IV criteria diagnosis (reflected also in the recently published DSM-5 criteria). ${ }^{20}$ Attention is a basic component of cognitive function, and can affect performance in many other cognitive domains. It is affected in many disorders other than delirium, including dementia, depression and developmental conditions, such as attention-deficit hyperactivity disorder. Other factors which may impact negatively on measures of attention are level of education, female sex and increasing age, ${ }^{21-23}$ however reports are conflicting. ${ }^{22} 2425$ Attention is particularly affected in Dementia with Lewy Bodies (DLB), which is phenomenologically and neurochemically more similar to delirium than other dementias. ${ }^{26}$ In Alzheimer's dementia (AD), complex attentional functions are affected early on in the disease with performance on more basic attention tasks relatively preserved until the advanced stages of the condition, ${ }^{27}$ however, it is thought that the deficits may be reflective of more primary memory problems. ${ }^{28}$ Contrastingly, in delirium, the attentional deficit is more global, more marked, and being a mandatory feature of delirium, occurs with much higher frequency than other cognitive deficits. ${ }^{29}$ Bedside tests to capture inattention are simple and quick to perform, and include 'WORLD backwards' and 'serial 7s' from Folstein's Mini Mental State Examination (MMSE) ${ }^{30}$ and reciting the months of the year or the days of the week backwards. ${ }^{3132}$ The former two tests from the MMSE are well recognised to be particularly sensitive to educational level. ${ }^{33}$ Other examples, such as the Digit Span Test, the Vigilance 'A' Test, and the Digit Cancellation Test have been shown to aid delirium detection. $^{3435}$ The visual Spatial Span Forwards (SSF), a pattern recognition test based on the digit span forwards, ${ }^{36}$ has recently shown to be of some use in identifying inattention in patients with delirium versus those with dementia. ${ }^{37}$ These bedside tests are somewhat observer-dependent, and may be affected by deficits in other domains, such as visual or auditory processing speed and motor execution. The Edinburgh Delirium Test Box is a device which was developed specifically to objectively measure performance on tasks of sustained visual attention only. Using this device, Brown and colleagues showed that patients with delirium performed much more poorly on sustained attention tasks than those with $\mathrm{AD}$ or healthy controls. Additionally, the device showed excellent accuracy for discriminating delirium from dementia and cognitively intact controls. ${ }^{38}$ Using devices such as this, however, is cumbersome and, hence, we considered simple bedside tests, which require minimal training, to be more feasible for routine and repeated use in a busy clinical setting. Thus, the aim of our study was to determine if the simple bedside attention tests, Months of the Year Backwards (MOTYB) and SSF, or reports of confusion (either subjective or objective) were predictive of the presence of delirium. We also aimed to assess the usefulness of the CAM, as a second-line screening step following initial testing.

\section{METHODS}

This study was part of a large point-prevalence study of delirium, which was conducted at Cork University Hospital (CUH) on 15th May 2010. The details of this study's methodology and ethical procedures have been published elsewhere. ${ }^{1}$ All adult inpatients were eligible for inclusion in the study, excluding those in the ED, intensive care unit and haematology/burns isolation unit. Patients were also excluded if they refused participation, or were severely aphasic; comatose; dying; or considered too unwell for interview by nursing staff.

The study involved three stages of assessment: attention testing/screening for subjective or objective 'confusion'; CAM assessment; and formal evaluation by experienced psychiatrists. Trained junior medical staff first screened every patient for inattention using the SSF and MOTYB. The SSF was performed using an A5-sized piece of white card with eight red squares (each measuring $1.5 \mathrm{~cm}^{2}$ ) evenly spaced over three rows (configuration three, two, three; landscape; see online supplementary files). The investigators tapped out predetermined sequences for the patients to replicate. The test began with a sequence of two squares and increased in number with each correct iteration, up to a maximum sequence of seven. Two attempts were allowed at each level using different predetermined sequences. Patients who were unable to correctly repeat a sequence of five were considered to have failed the test. For the MOTYB, the patients were first requested to say the months of the year forward from January to December. They were then asked to recite the months in reverse order from December back to January. Patients were considered to have passed this test on reaching July without error. Hence, inattention was deemed present in those who scored less than five on SSF, or were unable to correctly recite the MOTYB as far as July. Additionally, the patient was screened for subjective confusion, and objective reports of confusion (or proxy terms) by nursing staff interview and inspection of the medical notes. The patients were asked the following question: 'Have you felt muddled in your thinking, or confused, since you came into hospital?' to determine the presence of subjective confusion. The nurses were interviewed using a standardised set of questions (available as an online supplementary file and published elsewhere ${ }^{1}$ ), used to investigate the presence or absence of delirium features. The medical notes were also searched for any documentation of delirium or the proxy terms 'confusion' or 'agitation' during the admission. The patient was considered to have objective evidence of confusion if nursing staff responded positively to any of the nursing questions relating to delirium, and/or if there was reference to delirium or proxy term in the medical notes.

Patients who failed at least one of the attention tests, or who had subjective or objective reports of confusion, were then independently assessed using the sensitive, short form of the CAM, by a team of eight trained Geriatric Medicine registrars and consultants, ${ }^{39}$ and deemed either CAM-positive or CAM-negative. These doctors had undergone $8 \mathrm{~h}$ of CAM training over a 3-month period, including instruction, discussion, simulated cases, interval online self-assessment and, finally, trainerobserved CAM performance and scoring with feedback. Table 1 illustrates how each item on the CAM was scored. Of note, the SSF and MOTYB were repeated by the CAM assessors for the purposes of scoring the CAM.

Patients who underwent CAM then proceeded to be assessed by a team of four experienced psychiatrists, with specific expertise in delirium detection, using the Delirium Rating ScaleRevised '98 (DRS-R98) ${ }^{40}$ (time to psychiatry assessment 
Table 1 Details of how each item on the CAM was scored by the CAM assessors

\section{Example of how the CAM was performed}

1. Introduction: "Hello, Mr.... My name is .... As you have been informed, a study is ongoing in the hospital today and earlier you agreed to participate. You have already answered some questions for us. Are you happy for me to ask you some more questions as part of the same study? It should not take longer than five minutes."

2. General assessment: General conversation questions to assess if any obvious distractibility or disorganised thinking (eg, how are you feeling today?; how long have you been in hospital?; etc.)

3. Formal assessment: Testing of attention and thought process using methods described below.

4. Nursing questionnaire: Responses from screening nursing questionnaire viewed for information relating to temporal onset and fluctuations

\section{CAM item}

1A: Acute onset

1B: Fluctuating course

2: Inattention

3: Disorganised thinking

\section{How each item was scored}

This was scored using answers from the standardised nursing interview.

The raters observed for evidence of fluctuations during the CAM interview. The standardised nursing interview was also used to assess for presence of fluctuations.

The SSF and MOTYB were repeated by the CAM assessors. This item was positive if either one of the tests was failed on this occasion, or if there was evidence of inattention or distractibility during the interview. The results from the initial screening attention tests were not used.

The patient was asked the following questions:

- Can you tell me what this proverb means? 'Every cloud has a silver lining' (example)

- Abstract questions*:

1. Would a stone float on water?

2. Would two pounds of flour weigh more than one pound?

Disorganised thinking was considered present if the patient was unable to correctly interpret the proverb or answered either of the abstract questions incorrectly, or if the patient demonstrated obvious evidence of disorganised thinking during the interview.

4: Altered level of consciousness This item was considered positive if there was any evidence of drowsiness/hyperalertness during the interview.

*From the CAM-ICU. ${ }^{17}$

CAM, Confusion Assessment Method; MOTYB, Months of the Year backwards; SSF, Spatial Span Forwards.

$<24 \mathrm{~h}$ ). This is a well-validated, ${ }^{40-42}$ diagnostically precise tool which displays high inter-rater reliability, validity, sensitivity and specificity for differentiating delirium from mixed neuropsychiatric conditions including dementia and depression. ${ }^{40} 4243$ This assessment was performed completely independently of the two previous stages, and all items were scored according to the DRS-R98 training manual. ${ }^{44}$ The presence of delirium was ultimately determined according to DSM-IV criteria (reference standard).

\section{Assessment of previous cognitive status}

In all patients with delirium, the medical case-notes were reviewed for a diagnosis of pre-existing dementia made by a suitably trained physician. Where this was not available, premorbid cognition was determined by telephone interview using the Informant Questionnaire on Cognitive Decline in the Elderly-Short Form (IQCODE-SF), a validated screening tool for detecting dementia. ${ }^{45}$ Patients without delirium who were less than 65 years of age were presumed not to have dementia unless it was documented in the case notes. As dementia is more prevalent and is known to be underdetected in older people, depending on medical chart documentation for diagnosis was likely to be highly undersensitive. Hence, a random sample of 40 older non-delirious patients also had baseline preadmission cognition assessed using the IQCODE-SF.

\section{Other data collected}

Information relating to medication use was documented, and laboratory results (sodium, glucose, thyroid stimulating hormone, calcium, urea, C-reactive protein, white cell count and albumin level) were also collected. Current and previous alcohol history was recorded where available, and the Charlson comorbidity index ${ }^{47}$ was calculated.

\section{Statistical analyses}

Statistical analysis was performed using SPSS V.18. To compare baseline characteristics between patients with and without delirium, Mann-Whitney U-test was used. Sensitivities and specificities were calculated for each screening test/combination of tests from $2 \times 2$ tables, with CIs testing significance at 95\%. Each screening method was assessed in this way initially in isolation, and then subsequently in combination with other screening methods. Hence, we examined the performance of a variety of test combinations. This included applying test combinations simultaneously or sequentially, and then with or without the CAM as a further screening step. There are two potential simultaneous test combination scenarios: (1) screen positive if at least one test failed (figure 1A) and (2) screen positive if both tests failed (figure 1B). These two approaches were analysed separately. In sequential test combinations, the second test is only applied in the setting of a failed first test (figure 1C). Simultaneous testing in which a patient screens positive if either or both screening methods are failed (scenario 1) will always have a higher net sensitivity than either sequential tests or simultaneous tests where both tests must be failed to screen positive (scenario 2); however, there is a net loss in specificity. Sequential testing results in a net loss in sensitivity, but a net gain in specificity. ${ }^{48}$

A modified version of the Forest Plot viewer programme was used to create graphical representation of the data. ${ }^{49}$ As advancing age and prior history of dementia have previously been shown to be independent predictors of delirium in this cohort, ${ }^{1}$ we repeated all calculations in those older than and younger than the median age of 69 years, and in those with and without a previous history of dementia. In total, over 70 test combinations were analysed.

\section{RESULTS}

There were 311 patients included in the delirium pointprevalence study and 280 had full data available, 55 of whom 


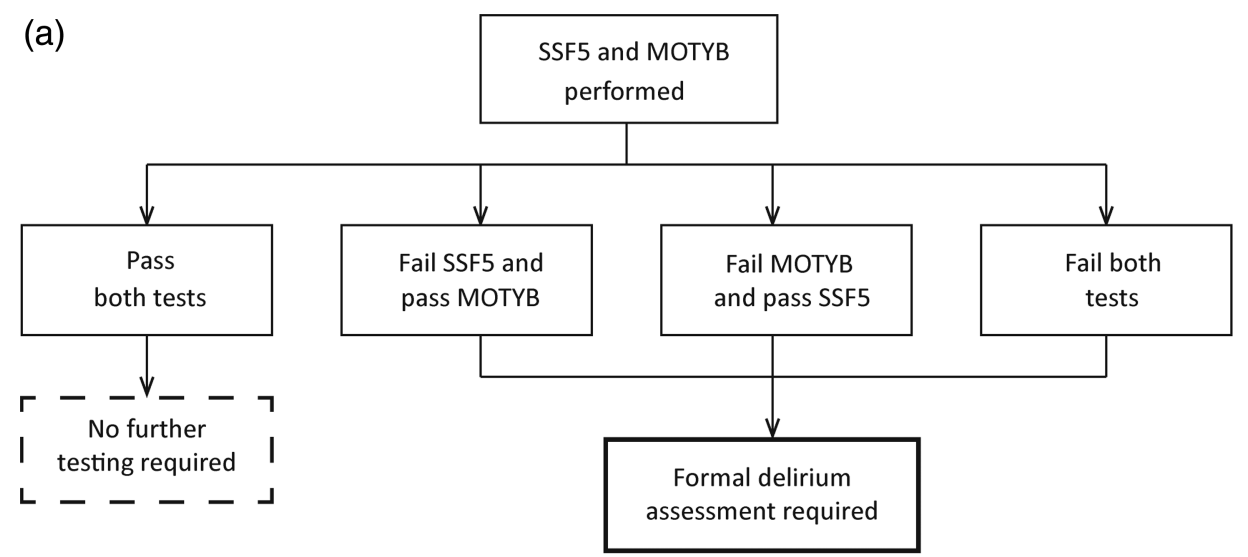

(b)
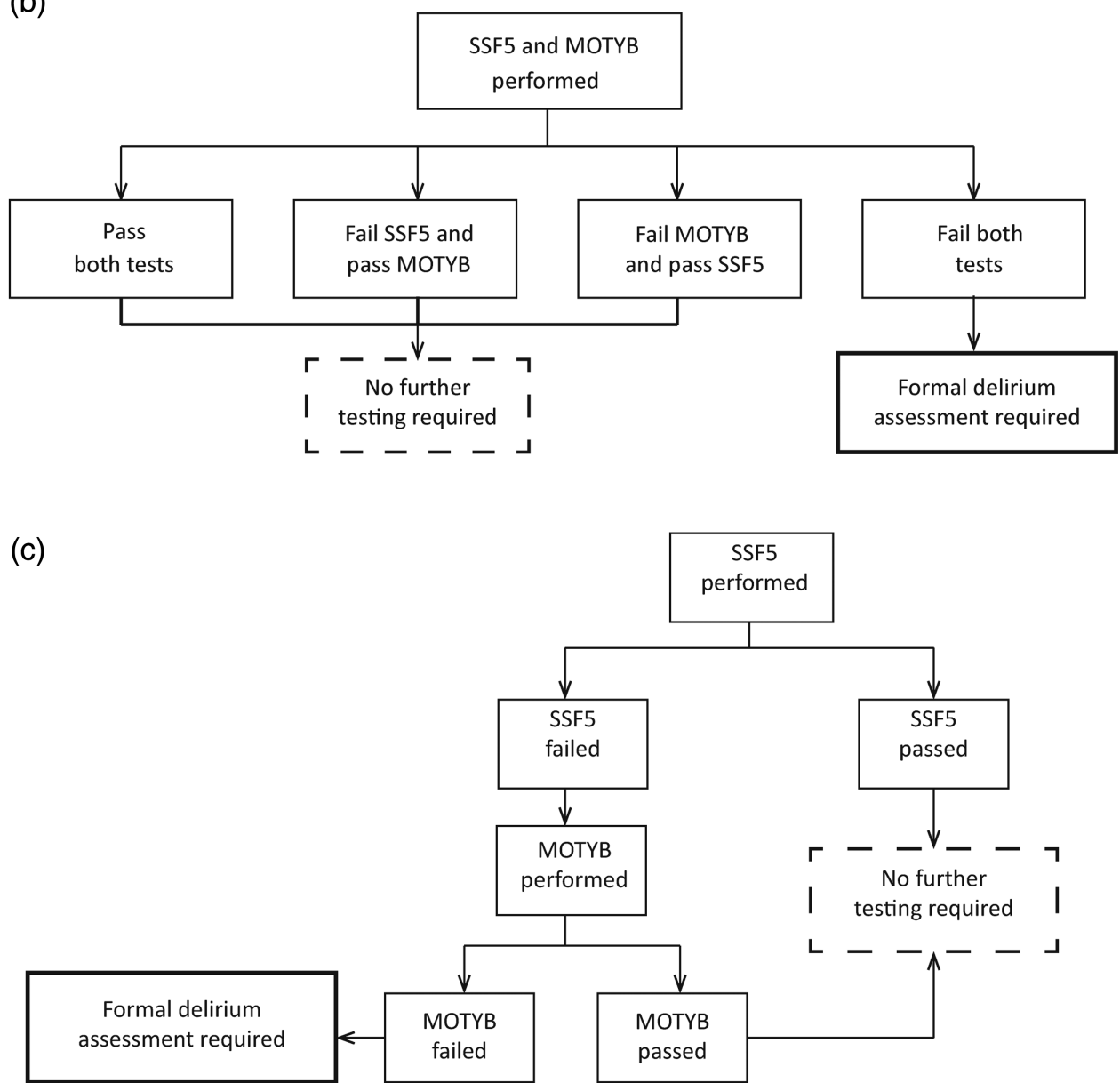

Figure 1 Testing processes using different test combination models a) Example of simultaneous testing (a): Two screening tests administered simultaneously. Further assessment required if either test failed. b) Example of simultaneous testing (b): Two screening tests administered simultaneously. Further assessment required only if both tests failed c) Example of sequential testing: First screening test is performed. Proceed to second screening test only if first test failed. Then proceed to further assessment only if second test is also failed. (MOTYB=months of the year backwards test; SSF5=spatial span forwards test with a cutoff of 5).

were diagnosed with DSM-IV delirium (19.6\%). ${ }^{1}$ For this analysis, 265 patients were included, 48 (18.1\%) of whom had DSM-IV delirium. We excluded those who had not completed SSF and MOTYB testing (hence, excluding patients with severe visual difficulties and aphasia), and those who had not had subjective and/or objective 'confusion' status recorded (see figure 2). Patients who were deemed to have objective evidence of confusion are those who were reported as confused by nursing staff and/or had documentation of confusion in the medical notes. Of the included patients, 23 had no data pertaining to medical documentation of delirium and four had missing data in relation to nursing opinion. Patient demographics for this cohort are outlined in table 2 . The median age of the cohort was 69 years (range 17-95) and 51.1\% were men. Reason for admission was recorded in $84.9 \%(n=225)$ included patients. The most commonly documented reasons for admission were, first, to 


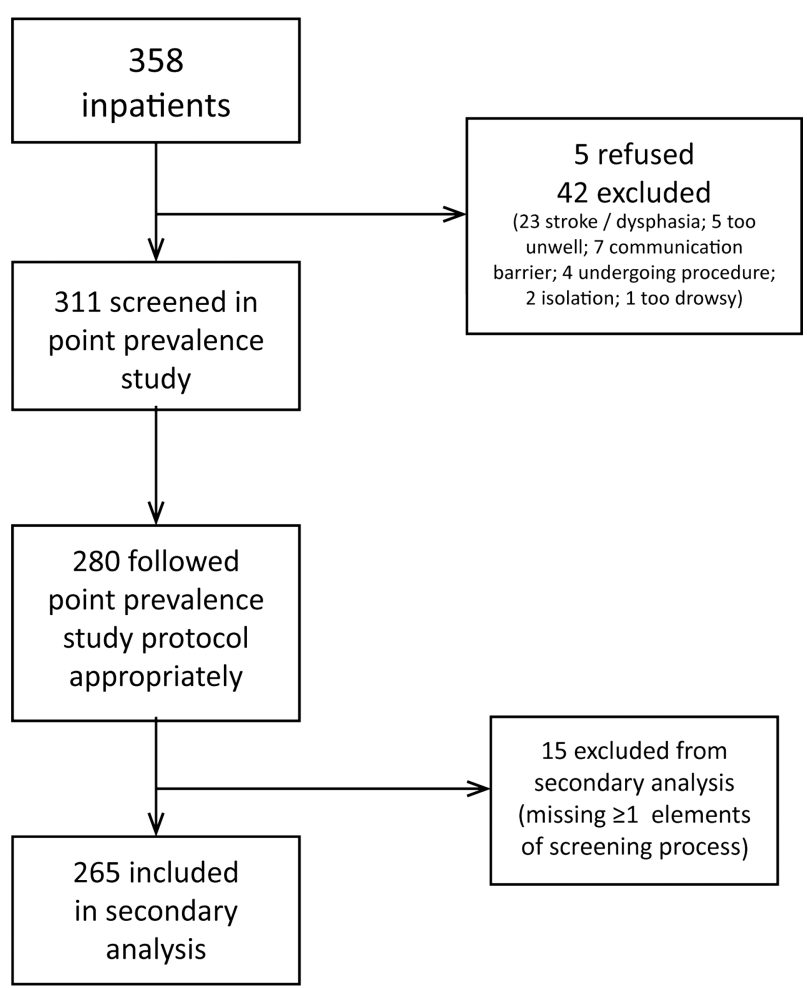

Figure 2 Flow of patients through the study.

undergo procedure/surgery $(n=39,14.7 \%)$, followed by neurological causes $(n=33,12.5 \%)$, respiratory causes $(n=31$, $11.7 \%)$, cardiac causes $(n=30,11.3 \%)$ and malignancy/tumour $(\mathrm{n}=25,9.4 \%)$.

Figure 3A illustrates the sensitivity, specificity and 95\% CIs for each screening test individually, and additionally with a subsequent CAM assessment, in the prediction of delirium for the whole group. The most accurate single test was MOTYB, with a sensitivity of $83.3 \%$ (95\% CI 69.8 to 92.5 ) and specificity of $90.8 \%$ (95\% CI 86.1 to 94.3 ). SSF5 (SSF using a cutoff of 5: ie, a patient who can repeat a maximum sequence of four squares has inattention) was highly sensitive $(91.7 \%$, 95\% CI 80 to 97.6) but lacked specificity $(69.12 \%, 95 \%$ CI 62.5 to 75.2$)$. However, SSF4 (using a cutoff of 4) missed almost one-quarter of delirium cases (sensitivity 77.1\%, 95\% CI 62.7 to 87.9). Figure $3 \mathrm{~A}$ also illustrates that although the addition of the CAM as a second-line screening step predictably increased net specificity of each method, it led to considerable net loss in sensitivity. For example, of 265 included patients, 60 patients failed the

Table 2 Patient demographics

\begin{tabular}{|c|c|c|c|c|}
\hline & $\begin{array}{l}\text { Total } \\
(n=265)\end{array}$ & $\begin{array}{l}\text { Delirium } \\
(n=48)\end{array}$ & $\begin{array}{l}\text { No } \\
\text { delirium } \\
(\mathrm{n}=217)\end{array}$ & Sig. \\
\hline Age (years), median (IQR) & $69(27)$ & $78(15.25)$ & 66 (29.5) & $\mathrm{p}<0.001$ * \\
\hline Sex (\% male) & 51.1 & 52.1 & 50.7 & $p=0.872 \dagger$ \\
\hline \multicolumn{5}{|l|}{ Dementia status $(n=194)$} \\
\hline Dementia, $\mathrm{n}(\%)^{\ddagger}$ & $31(16.0)$ & $24(50.0)$ & $7(4.8)$ & $p<0.001 \neq$ \\
\hline $\begin{array}{l}\text { *Independent Samples Man } \\
\text { tFisher's Exact test. } \\
\text { ₹Number of patients with } d \\
\text { total number of patients in } \\
(n=194) \text {; delirium }(n=48) ; n\end{array}$ & $\begin{array}{l}\text { nitney } U \text { tes } \\
\text { tia in each } \\
\text { group in } w ! \\
\text { rium }(n=14\end{array}$ & $\begin{array}{l}\text { oup is pres } \\
\mathrm{m} \text { dementi }\end{array}$ & $\begin{array}{l}\text { ed as a } \\
\text { atus wa }\end{array}$ & $\begin{array}{l}\text { tage of } \\
\text { wn: total }\end{array}$ \\
\hline
\end{tabular}

MOTYB and proceeded to CAM assessment. Of these, 40 patients were subsequently diagnosed with DSM-IV delirium, 10 of whom were missed by the CAM, hence a sensitivity of $75 \%$. Of the 20 patients without DSM-IV delirium, only three had been CAM-positive, yielding a specificity of $85 \%$.

Following assessment of each individual test, we analysed the performance of a variety of test combinations, as described earlier. Figure 3B illustrates the most efficient test combinations for the prediction of delirium for the overall group (additional data in online supplementary files). Using simultaneous approach (scenario 1) appears most effective, in particular the combination labelled 'CONF/MOTYB', which means that the patient failed MOTYB, or was confused subjectively/objectively (CONF). This combination had a sensitivity of $93.8 \%(95 \%$ CI 82.8 to 98.6 ) and a specificity of $84.7 \%$ (95\% CI 79.2 to 89.2 ) and was marginally more accurate than the simultaneous application (scenario 1) of MOTYB and SSF4, sensitivity of $93.8 \%$ (95\% CI 82.8 to 98.6 ); specificity $81.1 \%$ (95\% CI 75.2 to 86.1).

As mentioned earlier, analysis of test combinations was also performed in older and younger patients. Figures representing this data are available as supplementary material. The MOTYB as a single test was best for those over the median age of 69 years, having high sensitivity and specificity for prediction of delirium, 83.8\% (95\% CI 68 to 93.8) and 89.6\% (95\% CI 81.7 to 94.9 ). Contrastingly, for younger patients, many test options were highly accurate, the most precise being a simultaneous (scenario 1) combination of SSF4 and subjective/objective confusion (sensitivity $100 \%, 95 \%$ CI 73.3 to 100 ; specificity $87.5 \%$, 95\% CI 80.2 to 92.8 ) or SSF (cut-off 4 ) and MOTYB (sensitivity $100 \%, 95 \%$ CI 71.3 to 100 ; specificity $86.8 \%$, 95\% CI 79.4 to 92.2). In this younger subgroup, evidence of confusion alone, without the use of any attention test, was also highly predictive of delirium (sensitivity $90.9 \%$, 95\% CI 58.7 to 98.5 ; specificity 92.5\%, 95\% CI 86.2 to 96.5 ).

Pre-existing cognitive status, as outlined above, was ascertained in 194 patients. Four patients had a documented history of dementia in the medical notes, all of whom had delirium. A further 27 were considered to have pre-existing dementia based on an IQCODE-SF of $\geq 3.5$. Prior cognitive impairment independently predicted a diagnosis of delirium (adjusted OR 15.3; CI 5.2 to $45.4, \mathrm{p}<0.001) .{ }^{1}$ In patients with dementia, MOTYB was again the most sensitive single test $(87.5 \%, 95 \%$ CI 67.6 to 97.2), however the specificity was low and did not reach statistical significance $(71.4 \%, 95 \%$ CI 29.3 to 95.5$)$. In patients without dementia, three screening combinations had almost equivalent accuracy. Using MOTYB and SSF4, scenario (a), picked up $87.5 \%$ (95\% CI 67.6 to 97.2 ) of delirium cases, and was $86.3 \%$ (95\% CI 79.5 to 91.6 ) specific. Very similar results were achieved using either one of these tests simultaneously with seeking for evidence of 'confusion' (scenario 1). Table 3 displays a summary of the most diagnostically accurate approaches.

\section{DISCUSSION}

Delirium is vastly underdetected, a factor which contributes greatly to its long-term personal, social and economic burden. Most clinicians do not routinely screen for delirium in practice, possibly in part due to an underappreciation of its impact, but also due to a paucity of brief screening assessments. ${ }^{9}{ }^{50}$ Most of the currently employed assessment techniques take longer than $5 \mathrm{~min}$ to perform, ${ }^{51}$ for example, the CAM, which in addition, requires training for accurate use. ${ }^{18}$ Recently, Han et al found that using a two-step screening process based on a 
(a)

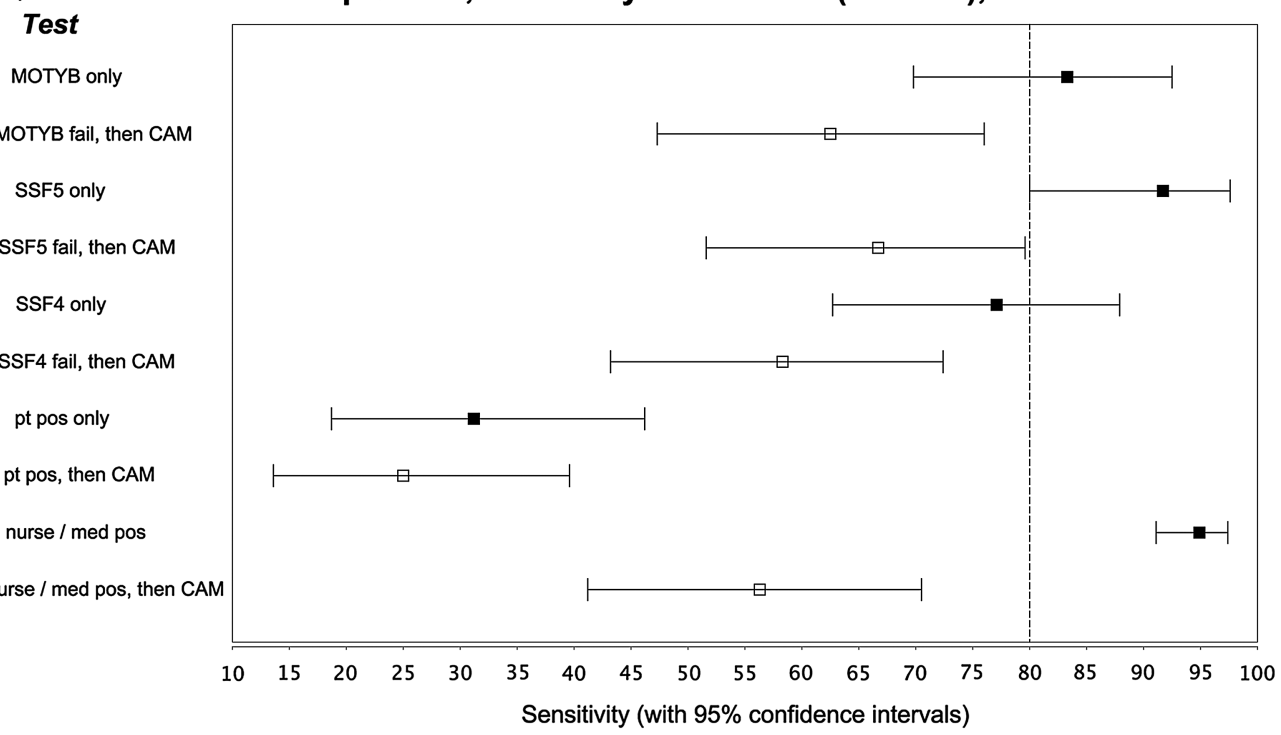

(b)

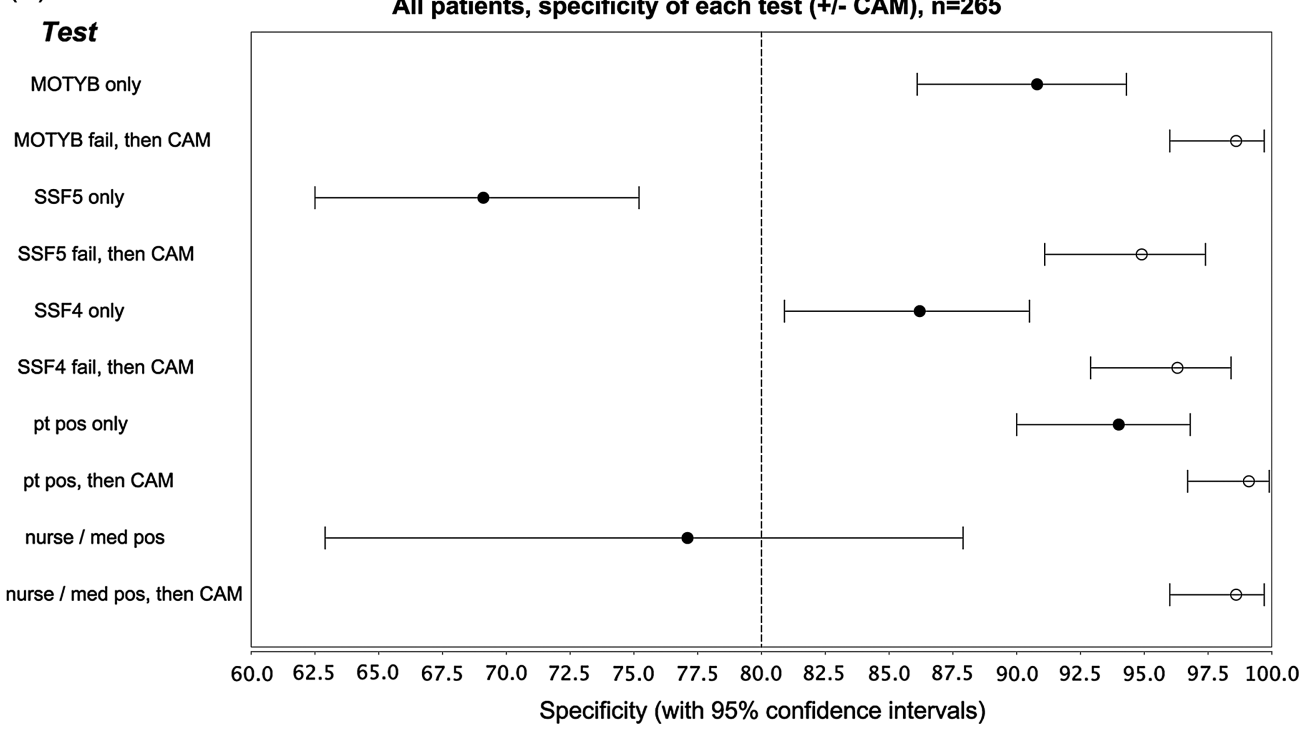

Figure 3 Forest plots depicting performance of tests individually and in combination: sensitivity and specificity plotted with $95 \%$ confidence intervals 3a) Sensitivity of each individual test, with and without the CAM as a second-line test 3b) Specificity of each individual test, with and without the CAM as a second-line test 3c) Most efficient test combinations, sensitivity 3d) Most efficient test combinations, specificity (MOTYB=Months of the year backwards; SSF5=SSF with cutoff of 5; SSF4= SSF with cutoff of 4; Pt pos=Subjective confusion (patient felt subjectively confused when questioned); Nurse pos=nurse thought patient was confused when questioned; Med pos='confusion' or proxy term documented in the patient's medical notes; Nurse or med pos=0bjective confusion (either nurse felt patient was confused or 'confusion' or proxy term was documented in the medical notes); Nurse/med pos=objective confusion (by nurse report and/or medical documentation); CONF/MOTYB pos=any evidence of confusion and/or MOTYB failed; CONF/SSF5 pos=any evidence of confusion and/or SSF failed with a cutoff of 5; CONF/SSF4 pos=Any evidence of confusion and/or SSF failed with a cutoff of 4; MOTYB/SSF5 pos=MOTYB failed and/or SSF5 failed with a cutoff of 5; MOTYB/ SSF4 pos=MOTYB failed and/or SSF failed with a cutoff of 4]

brief operationalised version of the CAM (b-CAM) in the ED, had a sensitivity of $70.0-84.0 \%$ and a specificity of $95.8-$ $97.2 \%$ when tested against DSM-IV diagnosis. ${ }^{52}$ The authors describe the b-CAM taking $<1$ min to perform, however, given that collateral history is required to assess temporal onset and fluctuations, it is likely that the process could often be more lengthy. In a similar vein, in this report, we describe the performance of a number of screening methods in the prediction of DSM-IV delirium across a large general hospital. We assessed three attention tests (MOTYB, SSF5, SSF4), as well as staff and patient's own impression of cognitive status. We examined the predictive potential of each screening method in isolation, followed by various screening combinations. Additionally, for each method, we assessed whether or not using the CAM as a second-line screening step improved accuracy. We also investigated if tailoring the approach depending on patient age, or prior cognitive status could improve efficiency.

The best overall individual screening test was MOTYB, with a sensitivity of $83.3 \%$ and a specificity of $90.8 \%$ for the entire group. The simultaneous addition of another screening method (either SSF4 or evidence of subjective/objective confusion), 
(c)

All patients, best tests (sensitivity), $\mathrm{n}=\mathbf{2 6 5}$

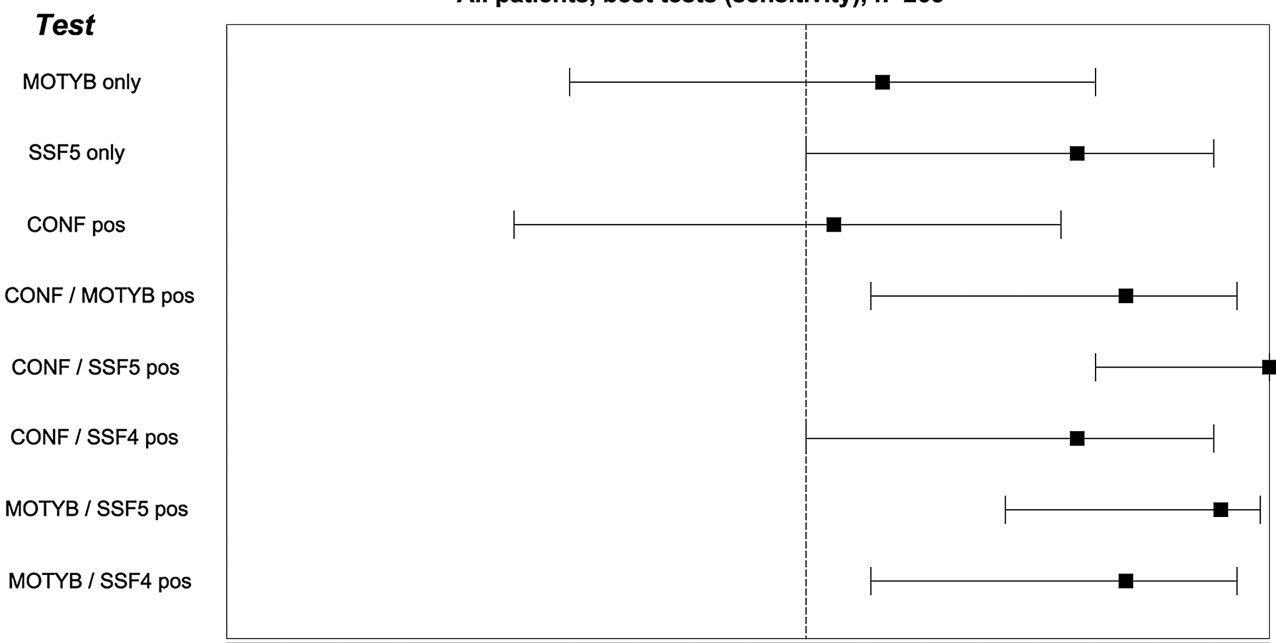

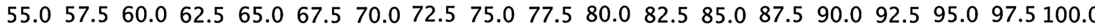

Sensitivity (with $95 \%$ confidence intervals)

(d)

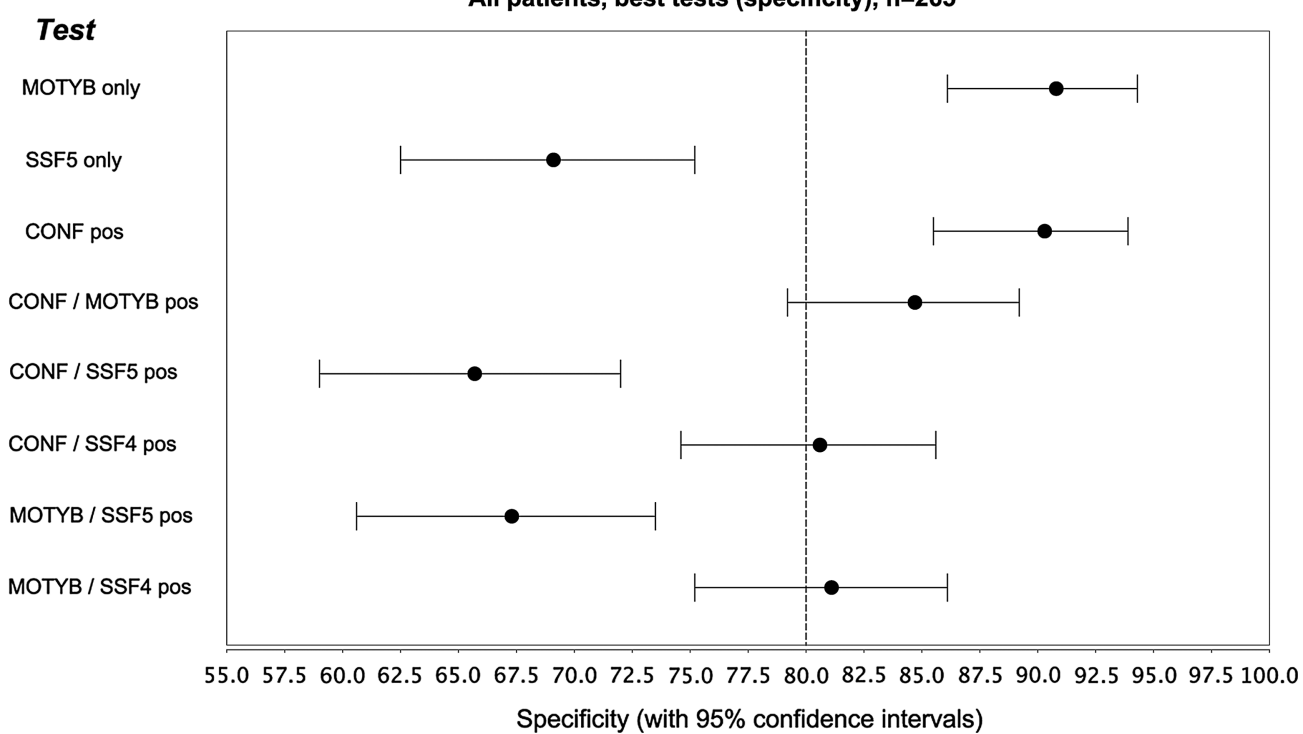

Figure 3 (Continued)

where any result being abnormal denoted a positive screen, increased net sensitivity to over $90 \%$. Subanalysis in patients over and under the median age of 69 years, and in patients with and without dementia, gave similar results. In all subgroups, MOTYB performed well as an individual screening test, and in older patients or those with dementia, this approach was best. In younger patients or those without dementia, using MOTYB as part of a two-pronged screening approach, rather than alone, increased sensitivity without compromising specificity. It is also interesting to note that in younger patients, any evidence of confusion was highly predictive of delirium. This implies that formal attention testing may be less crucial in this group, as any evidence of confusion raises a strong suspicion of delirium. The SSF is a quick, simple attention test, which can be used in patients with expressive language difficulties. Using a cutoff of 5 , it is a highly sensitive test for the presence of delirium, however, specificity is low at $69 \%$. When the cutoff is lowered to 4 , almost one-quarter of patients with delirium are missed. Nonetheless, this remains more accurate than staff detection (nursing staff missed one-third of cases, and medical staff failed to recognise one-half). ${ }^{1}$ These results are also consistent with previous work which illustrated that lower scores on the SSF are highly predictive of delirium, but higher scores are less useful in outruling its presence. ${ }^{37}$ Our study shows that the SSF appears to be particularly useful as a single test in patients with no prior history of dementia, when a cut-off of 5 yields a sensitivity of 91.7\% and specificity of $75.5 \%$.

As mentioned earlier, deficits in sustained attention occur in the late stages of $\mathrm{AD}$, but performance on more complex attentional functions is impaired earlier in the disease. ${ }^{27}$ Some studies have used the MOTYB to distinguish stages of $\mathrm{AD}, 5354$ including one small study which showed that the predictive value of the MMSE for dementia could be augmented by the addition of the MOTYB. ${ }^{53}$ In a study of multiple cognitive domains in patients with $\mathrm{AD}$ and fronto-temporal dementia (FTD), performance on Digit Span Forwards and Backwards and on Vigilance 'A' and 'B' tests was impaired to varying degrees in typical AD and FTD when compared with normal 
Table 3 Most accurate screening methods from our study, overall group and subgroups based on age and cognitive status. Our preferred screening approaches highlighted in bold

\begin{tabular}{|c|c|c|c|c|}
\hline & & Screening method & $\begin{array}{l}\text { Sensitivity* } \\
(95 \% \mathrm{Cl})\end{array}$ & $\begin{array}{l}\text { Specificity* } \\
(95 \% \mathrm{Cl})\end{array}$ \\
\hline General hospital inpatients $(n=265)$ & $\begin{array}{l}\text { Single test } \\
\text { Simultaneous tests }\end{array}$ & $\begin{array}{l}\text { MOTYB } \\
\text { MOTYB/evidence of confusion (either positive = positive) } \\
\text { MOTYB/SSF4 (either failed = positive) }\end{array}$ & $\begin{array}{l}83.3 \%(69.8-92.5) \\
93.8 \%(82.8-98.6) \\
93.8 \%(82.8-98.6)\end{array}$ & $\begin{array}{l}90.8 \%(86.1-94.3) \\
84.7 \%(79.2-89.2) \\
81.1 \%(75.2-86.1)\end{array}$ \\
\hline Older inpatients, $\geq 69$ years ( $n=133$ ) & Single test & MOTYB & $83.8 \%(68-93.8)$ & $89.6 \%(81.7-94.9)$ \\
\hline Younger inpatients, $\leq 69$ years $(n=132)$ & $\begin{array}{l}\text { Single test } \\
\text { Simultaneous tests }\end{array}$ & $\begin{array}{l}\text { Evidence of confusion } \\
\text { SSF4/evidence of confusion (either positive = positive) } \\
\text { SSF4/MOTYB (either positive = positive) }\end{array}$ & $\begin{array}{l}90.9 \%(58.7-98.5) \\
100 \%(73.3-100) \\
100 \%(73.3-100)\end{array}$ & $\begin{array}{l}92.5 \%(86.2-96.5) \\
87.5 \%(80.2-92.8) \\
86.8 \%(79.4-92.2)\end{array}$ \\
\hline Patients with known dementia $(n=31)$ & Single test & МотYв & $87.5 \%(67.6-97.2)$ & $71.4 \%(29.3-95.5)$ \\
\hline Patients with no history of dementia ( $n=154)$ & Simultaneous tests & MOTYB/SSF4 (either positive = positive) & $87.5 \%(67.6-97.2)$ & $86.3 \%(79.5-91.6)$ \\
\hline
\end{tabular}

*Sensitivities and specificities with $95 \%$ Cls based only on results from our study.

MOTYB, months of the year backwards test; ssf4, spatial span forwards with a cut-off of 4 .

controls, whereas patients with amnestic $\mathrm{AD}$ were less affected. ${ }^{55}$ Interestingly, studies using more objective computerised attention tests have found that AD patients do not demonstrate impairments in focusing ${ }^{56}$ or sustained attention, ${ }^{38}$ whereas delirious patients are significantly impaired in this domain. $^{38}$

In our study, although the SSF missed very few delirium cases in patients with dementia, even with a cut-off of 4 , its specificity was below 50\%. This indicates that the test is sensitive to cognitive impairment in general, and test failure may, in fact, be reflective of attentional deficits related to underlying dementia rather than delirium. MOTYB seemed more accurate at predicting delirium in the dementia group, however, results did not reach statistical significance, possibly due to small patient number (total $n=31$ ). Additionally, the low number of dementia patients who did not have delirium was very small $(n=7)$ and, hence, interpretation of specificity in this subgroup is difficult. In practice, it is often challenging to ascertain premorbid cognition in patients who are acutely unwell, and who may have associated delirium or subsyndromal delirium, especially without a readily available, accurate collateral history. Hence, using different screening approaches depending on prior cognitive status may not be as easily applicable as, for example, varying the approach based on age, and supports the use of more versatile tools, such as MOTYB.

Our study has some limitations. Ideally, formal delirium testing should have been performed on all included patients, however, due to the time-consuming nature of thorough delirium assessment, it would not have been feasible to perform 265 such assessments over the course of 1 day. Hence, only those who had an indication of possible delirium, as detailed earlier, were formally assessed. It is possible that a patient with delirium may have passed both attention tests during a lucid interval, and had no other suggestion of possible delirium (ie, no subjective confusion or recollection of confusion during the lucid period, and no staff reports of potential delirium features). This is an unlikely scenario, thus, it can be assumed that those patients without formal delirium assessment did not have delirium. To manage time constraints and to ensure feasibility, we were unable to examine all existing bedside attention tests. Some other well-known tests of attention were not tested, for example, spelling the word WORLD backwards; subtracting

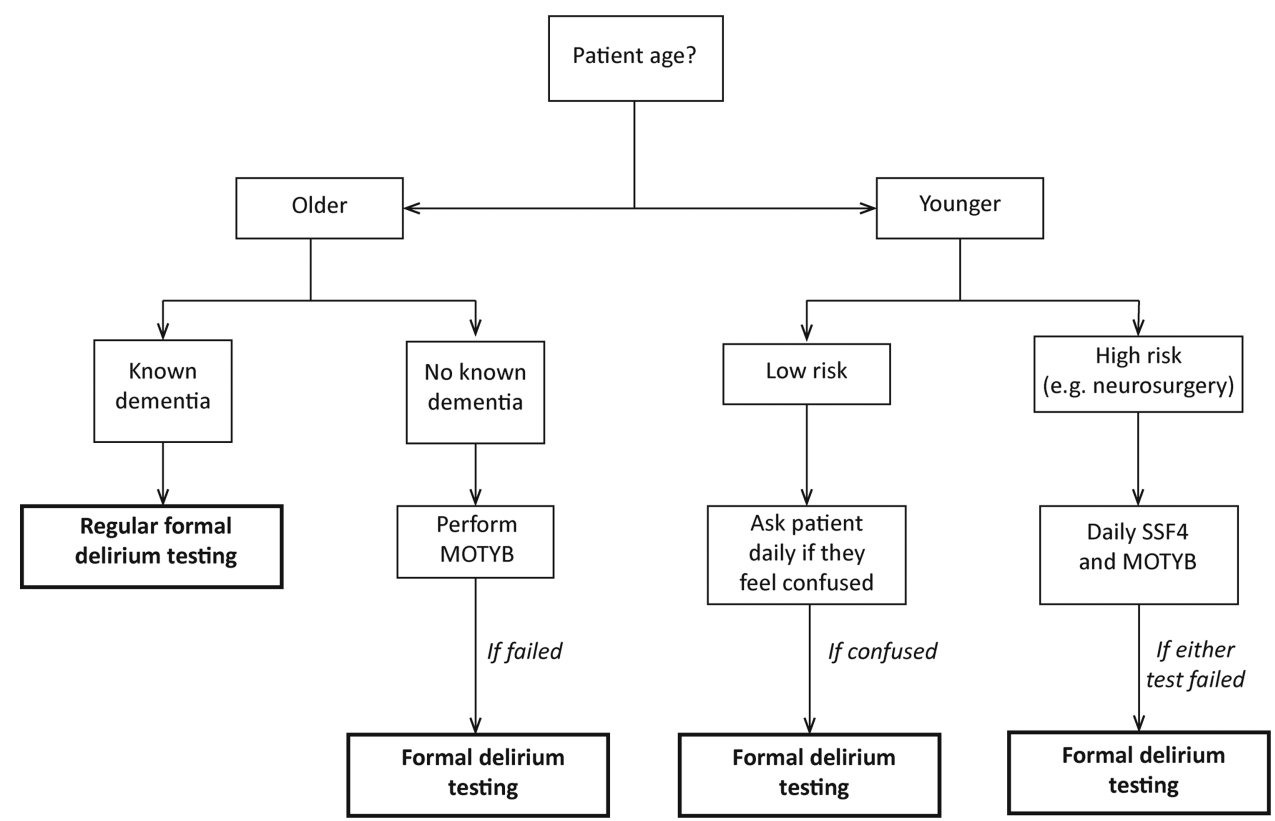

Figure 4 A suggested approach to delirium screening in the acute hospital setting (MOTYB;months of the year backwards test; SSF4;spatial span forwards test with a cutoff of 4 ). 
serial sevens from 100; and counting backwards from 20 to 1. The former two, taken from the MMSE (Mini-Mental State Examination), ${ }^{30}$ rely heavily on level of education and mathematical ability ${ }^{57}$ and, hence, were thought not to be suitable in this cohort. Counting backwards from 20 to 1 is taken from the Abbreviated Mental Test Score developed by Hodkinson. ${ }^{58}$ This test seemed too straightforward for inclusion and likely to have a considerable ceiling effect. Therefore, it seemed more logical to us to use a more challenging test, such as the SSF. As mentioned above, due to feasibility, we did not use objective computerised tests of attention.

In all test scenarios, the addition of the CAM as a second-line screening step, before proceeding to full delirium assessment, resulted in reduced net sensitivity. Other studies have shown low CAM sensitivity when used by inexperienced and minimally trained raters. ${ }^{18}$ In this study, all those performing CAM ratings underwent rigorous training in its use, based on the CAM training manual. ${ }^{39}$ One of the cornerstones of CAM assessment is accurate, dependable collateral history and, in this study, the temporal nature and evidence of fluctuations for the purposes of CAM scoring was based mainly on collateral history from on-duty nursing staff. Poor awareness of delirium features among staff, or a lack of emphasis during staff handover, can hinder attempts to ascertain their presence or absence during CAM assessment and make it extremely challenging to pinpoint acuity of onset or degree of fluctuations. We believe that this issue was a major contributor to the reduced sensitivity of the CAM in this study. The difficulty in obtaining accurate collateral history is a well-recognised barrier to delirium recognition in practice.

Our study involved a single sample of hospital inpatients, and further studies are required to extrapolate our findings to other samples and populations. Nonetheless, we conclude that simple tests of attention may provide an efficient method of screening for delirium in the acute hospital setting. Our suggested approach towards screening for each patient subgroup is illustrated in figure 4. It is well established that the hospitalised elderly are very delirium prone, and our study suggests that using MOTYB may be particularly useful for screening in this population. In younger people at risk of delirium, using a combination of MOTYB and SSF4, where failing either test is a positive screen, is more sensitive. The SSF, being a non-verbal attention test, has greater coverage in populations with speech or communication deficits, for example, stroke patients with dysphasia (also at high risk of delirium). In patients with known cognitive impairment, screening using the methods outlined may not be as effective, but this group is especially at risk $(77 \%$ in this study, but up to $89 \%$ in others ${ }^{59}$ ) and, hence, should undergo regular formal delirium testing during hospitalisation. In conclusion, we have described a novel, brief and straightforward screening approach for delirium detection across the general hospital. Our method requires minimal training and can be used by all healthcare professionals, including those with a limited understanding of delirium features. This brief approach to screening has the potential to greatly increase delirium recognition across the hospital, however, further prospective studies are needed to confirm our findings.

\footnotetext{
Author affiliations

${ }^{1}$ Centre for Gerontology and Rehabilitation, School of Medicine, University College Cork, Cork, Ireland

${ }^{2}$ Cork University Hospital, Cork, Ireland

${ }^{3}$ Department of Psychiatry, University of Limerick, Limerick, Ireland

${ }^{4}$ Department of Geriatric Medicine, Waterford Regional Hospital, Waterford, Ireland

${ }^{5}$ HRB Clinical Research Facility at UCC, University College Cork, Cork, Ireland
}

${ }^{6}$ Department of Psychiatry, University of Limerick, Limerick, Ireland ${ }^{7}$ Cognitive Impairment Research Group, Centre for Interventions in Infection, Inflammation \& Immunity (4i), Graduate Entry Medical School, University of Limerick, Limerick, Ireland

Acknowledgements We wish to thank the nursing and junior medical staff of Cork University Hospital who assisted with this study.

Contributors All authors meet ICJME criteria for authorship, and had full access to the data: All authors have substantial contributions to conception and design, acquisition of data, or analysis and interpretation of data; drafting the article or revising it critically for important intellectual content; and final approval of the version to be published. NAO, DJR, JC, DM and ST: all substantially contributed to study conception and design, data collection, statistical analysis and interpretation, drafting the initial manuscript, and subsequent critical revision of the manuscript and final approval. JAE: assisted with statistical analysis, contributed to drafting the original manuscript, performed critical revisions and has approved the final manuscript. EB, WC, CMG and ML: all majorly involved in data acquisition, critical revisions and final approval of the manuscript. The lead author, Dr Niamh O'Regan, affirms that the manuscript is an honest, accurate and transparent account of the study, and that no important aspects or discrepancies have been omitted.

Competing interests All authors have completed the ICJME uniform disclosure form at http://www.icjme.org/coi disclosure.pdf and declare: no support from any organisation for the submitted work; ST has received educational grants and honoraria from Janssen, Pfizer, Sanofi-Aventis, UCB, Astra-Zeneca, Orion, Lundbeck, Novartis, Boehinger, MSD pharmaceutical companies; no other relationships or activities that could appear to have influenced the submitted work.

Ethics approval This study was granted ethical approval by Cork Research Ethics Committee, Cork, Ireland.

Provenance and peer review Not commissioned; externally peer reviewed. Data sharing statement No additional data is available

Open Access This is an Open Access article distributed in accordance with the Creative Commons Attribution Non Commercial (CC BY-NC 3.0) license, which permits others to distribute, remix, adapt, build upon this work non-commercially, and license their derivative works on different terms, provided the original work is properly cited and the use is non-commercial. See: http://creativecommons.org/ licenses/by-nc/3.0/

\section{REFERENCES}

1 Ryan DJ, O'Regan N, Caoimh RO, et al. Delirium in an adult acute hospital population: predictors, prevalence and detection. BMJ Open 2013;3:e001772.

2 Trzepacz P, Meagher D, Leonard M. Delirium. In: Levenson J. ed. Textbook of Psychosomatic Medicine. 2nd edn. Washington, DC: American Psychiatric Publishing Press, 2010. Chapter 5, 71-114.

3 Kishi $Y$, Kato M, Okuyama $T$, et al. Delirium: patient characteristics that predict a missed diagnosis at psychiatric consultation. Gen Hosp Psychiatry 2007;29:442-5.

4 Cole MG. Delirium in elderly patients. Am J Geriatr Psychiatry 2004;12:7-21.

5 Inouye SK, Foreman MD, Mion LC, et al. Nurses' recognition of delirium and its symptoms: comparison of nurse and researcher ratings. Arch Intern Med 2001;161:2467-73.

6 Fang $\mathrm{CK}$, Chen HW, Liu SI, et al. Prevalence, detection and treatment of delirium in terminal cancer inpatients: a prospective survey. Jpn J Clin Oncol 2008;38:56-63.

7 Voyer $\mathrm{P}, \mathrm{McC}$ usker J, Cole MG, et al. Factors associated with delirium severity among older patients. J Clin Nurs 2007:16:819-31.

8 Collins N, Blanchard MR, Tookman A, et al. Detection of delirium in the acute hospital. Age Ageing 2010;39:131-5.

9 Han JH, Zimmerman EE, Cutler N, et al. Delirium in older emergency department patients: recognition, risk factors, and psychomotor subtypes. Acad Emerg Med 2009; 16:193-200.

10 Elie M, Rousseau F, Cole M, et al. Prevalence and detection of delirium in elderly emergency department patients. CMAJ 2000;163:977-81.

11 Milisen K, Lemiengre J, Braes T, et al. Multicomponent intervention strategies for managing delirium in hospitalized older people: systematic review. J Adv Nurs 2005;52:79-90

12 Gonzalez M, Martinez G, Calderon J, et al. Impact of delirium on short-term mortality in elderly inpatients: a prospective cohort study. Psychosomatics 2009:50:234-8

13 Kakuma R, du Fort GG, Arsenault L, et al. Delirium in older emergency department patients discharged home: effect on survival. J Am Geriatr Soc 2003:51:443-50.

14 Young J, Murthy L, Westby M, et al. Diagnosis, prevention, and management of delirium: summary of NICE guidance. BMJ 2010;341:c3704.

15 Inouye SK, van Dyck CH, Alessi CA, et al. Clarifying confusion: the confusion assessment method. A new method for detection of delirium. Ann Intern Med 1990:113:941-8. 
16 Wei LA, Fearing MA, Sternberg EJ, et al. The Confusion Assessment Method: a systematic review of current usage. J Am Geriatr Soc 2008;56:823-30.

17 Ely EW, Margolin R, Francis J, et al. Evaluation of delirium in critically ill patients: validation of the Confusion Assessment Method for the Intensive Care Unit (CAM-ICU). Crit Care Med 2001;29:1370-9.

18 Ryan K, Leonard M, Guerin S, et al. Validation of the confusion assessment method in the palliative care setting. Palliat Med 2009;23:40-5.

19 American Psychiatric Association. Diagnostic and statistical manual of mental disorders - text revision. 4th edn. Washington, DC: American Psychiatric Publishing Inc, 1994.

20 American Psychiatric Association. Diagnostic and statistical manual of mental disorders. 5th edn. Arlinglton, VA: American Psychiatric Publishing Inc, 2013.

21 Tun PA, Lachman ME. Age differences in reaction time and attention in a national telephone sample of adults: education, sex, and task complexity matter. Dev Psychol 2008;44:1421-9.

22 Gomez-Perez E, Ostrosky-Solis F. Attention and memory evaluation across the life span: heterogeneous effects of age and education. J Clin Exp Neuropsychol 2006;28:477-94

23 Mazaux JM, Dartigues JF, Letenneur L, et al. Visuo-spatial attention and psychomotor performance in elderly community residents: effects of age, gender, and education. J Clin Exp Neuropsychol 1995;17:71-81.

24 Van Gerven PW, Meijer WA, Jolles J. Education does not protect against age-related decline of switching focal attention in working memory. Brain Cogn 2007:64:158-63.

25 Chan RC. A further study on the sustained attention response to task (SART): the effect of age, gender and education. Brain Inj 2001:15:819-29.

26 Metzler-Baddeley C. A review of cognitive impairments in dementia with Lewy bodies relative to Alzheimer's disease and Parkinson's disease with dementia. Cortex 2007;43:583-600.

27 Kolanowski AM, Fick DM, Yevchak AM, et al. Pay attention! The critical importance of assessing attention in older adults with dementia. J Gerontol Nurs 2012;38:23-7.

28 Exton C, Leonard M. Eye tracking technology: a fresh approach in delirium assessment? Int Rev Psychiatry 2009:21:8-14.

29 Meagher DJ, Moran M, Raju B, et al. Phenomenology of delirium. Assessment of 100 adult cases using standardised measures. Br J Psychiatry 2007:190:135-41.

30 Folstein MF, Robins LN, Helzer JE. The mini-mental state examination. Arch Gen Psychiatry 1983:40:812

31 Katzman R, Brown T, Fuld $\mathrm{P}$, et al. Validation of a short Orientation-Memory-Concentration Test of cognitive impairment. Am J Psychiatry 1983;140:734-9.

32 Hall RJ, Meagher DJ, MacLullich AM. Delirium detection and monitoring outside the ICU. Best Pract Res Clin Anaesthesiol 2012;26:367-83.

33 Rosselli M, Tappen R, Williams C, et al. The relation of education and gender on the attention items of the Mini-Mental State Examination in Spanish speaking Hispanic elders. Arch Clin Neuropsychol 2006;21:677-86.

34 Pompei $\mathrm{P}$, Foreman $\mathrm{M}$, Cassel $\mathrm{CK}$, et al. Detecting delirium among hospitalized older patients. Arch Intern Med 1995:155:301-7.

35 O'Keeffe ST, Gosney MA. Assessing attentiveness in older hospital patients: global assessment versus tests of attention. J Am Geriatr Soc 1997:45:470-3.

36 Wilde NJ, Strauss E, Tulsky DS. Memory span on the Wechsler Scales. J Clin Exp Neuropsychol 2004;26:539-49.

37 Meagher DJ, Leonard M, Donnelly $\mathrm{S}$, et al. A comparison of neuropsychiatric and cognitive profiles in delirium, dementia, comorbid delirium-dementia and cognitively intact controls. J Neurol Neurosurg Psychiatry 2010;81:876-81.

38 Brown LJ, Fordyce C, Zaghdani H, et al. Detecting deficits of sustained visual attention in delirium. J Neurol Neurosurg Psychiatry 2011:82:1334-40.
39 Inouye SK. The confusion assessment (CAM): training manual and coding guide. New Haven, CT: Yale University School of Medicine, 1991

40 Trzepacz PT, Mittal D, Torres R, et al. Validation of the Delirium Rating Scale-revised-98: comparison with the delirium rating scale and the cognitive test for delirium. J Neuropsychiatry Clin Neurosci 2001:13:229-42.

41 Trzepacz PT, Dew MA. Further analyses of the delirium rating scale. Gen Hosp Psychiatry 1995;17:75-9.

42 Adamis D, Treloar A, MacDonald AJ, et al. Concurrent validity of two instruments (the Confusion Assessment Method and the Delirium Rating Scale) in the detection of delirium among older medical inpatients. Age Ageing 2005;34:72-5.

43 Trzepacz PT, Mulsant BH, Dew MA, et al. Is delirium different when it occurs in dementia? A study using the delirium rating scale. J Neuropsychiatry Clin Neurosci 1998; 10:199-204.

44 Trzepacz PT, Maldonado JR, Kean J, et al. Delirium Rating Scale-Revised-98 (DRS-R98) Administration Manual. A guide to increase understanding of how to solicit delirium symptoms when administering the DRS-R98., 2010.

45 Jorm AF. A short form of the Informant Questionnaire on Cognitive Decline in the Elderly (IQCODE): development and cross-validation. Psychol Med 1994;24: $145-53$

46 Jorm AF. The Informant Questionnaire on cognitive decline in the elderly (IQCODE): a review. Int Psychogeriatr 2004;16:275-93.

47 Charlson $M E$, Pompei $P$, Ales $K L$, et al. A new method of classifying prognostic comorbidity in longitudinal studies: development and validation. J Chronic Dis 1987:40:373-83.

48 Gordis L. Epidemiology. 3rd edn. Philadelphia, PA: Elsevier, 2004:71-94.

49 Boyles AL, Harris SF, Rooney AA, et al. Forest Plot Viewer: a new graphing tool. Epidemiology 2011:22:746-7.

50 Press Y, Margulin T, Grinshpun Y, et al. The diagnosis of delirium among elderly patients presenting to the emergency department of an acute hospital. Arch Gerontol Geriatr 2009:48:201-4.

51 Wong CL, Holroyd-Leduc J, Simel DL, et al. Does this patient have delirium?: value of bedside instruments. JAMA 2010;304:779-86.

52 Han JH, Wilson A, Vasilevskis EE, et al. Diagnosing delirium in older emergency department patients: validity and reliability of the delirium triage screen and the brief confusion assessment method. Ann Emerg Med 2013;62:457-65.

53 Tardiff M, Roy M, Bouchard R, et al. Months backwards test as a reliable predictor of cognitive decline in mild Alzheimer's disease. Alzheimers Demen 2013;9(4 supplement):P741-P42.

54 Ostberg P, Fernaeus SE, Bogdanovic N, et al. Word sequence production in cognitive decline: forward ever, backward never. Logoped Phoniatr Vocol 2008;33: 126-35.

55 Stopford CL, Thompson JC, Neary D, et al. Working memory, attention, and executive function in Alzheimer's disease and frontotemporal dementia. Cortex 2012;48:429-46

56 Ishizaki J, Meguro K, Nara N, et al. Impaired shifting of visuospatial attention in Alzheimer's disease as shown by the covert orienting paradigm: implications for visual construction disability. Behav Neurol 2013:26:121-9.

57 Ganguli M, Ratcliff G, Huff FJ, et al. Serial sevens versus world backwards: a comparison of the two measures of attention from the MMSE. J Geriatr Psychiatry Neurol 1990:3:203-7.

58 Hodkinson HM. Evaluation of a mental test score for assessment of menta impairment in the elderly. Age Ageing 1972;1:233-8.

59 Fick DM, Agostini JV, Inouye SK. Delirium superimposed on dementia: a systematic review. J Am Geriatr Soc 2002;50:1723-32. 